$\xi \mathrm{k}$

\title{
Determination of the predictive and prognostic values of polymorphism of some cell cycle genes in breast cancer
}

\author{
Samia A. Ebied ${ }^{1}$, Nadia A. Abd El Moneim ${ }^{2}$, Taha I. Hewala ${ }^{3 *}$, \\ Moustafa R. Abo Elsoud ${ }^{4}$, Gehan M. Shehata ${ }^{5}$, Heba Abass ${ }^{1}$ \\ ${ }^{1}$ Departments of Applied Medical Chemistry, Medical Research Institute, Alexandria University, \\ 165 El-Horria Avenue, El Hadara, Alexandria 21561, Egypt \\ ${ }^{2}$ Cancer Management and Research, Medical Research Institute, Alexandria University, \\ 165 El-Horria Avenue, El Hadara, Alexandria 21561, Egypt \\ ${ }^{3}$ Radiation Science, Medical Research Institute, Alexandria University, \\ 165 El-Horria Avenue, El Hadara, Alexandria 21561, Egypt \\ ${ }^{4}$ Experimental and Clinical Surgery, Medical Research Institute, Alexandria University, \\ 165 El-Horria Avenue, El Hadara, Alexandria 21561, Egypt \\ ${ }^{5}$ Bioinformatics and Medical Statistics, Medical Research Institute, Alexandria University, \\ 165 El-Horria Avenue, El Hadara, Alexandria 21561, Egypt \\ *Corresponding authorE-mail:tahahewala@hotmail.com
}

\begin{abstract}
Aim: To investigate the influence of the polymorphic variants of CCND1 (G870A) and p73 (G4C14- to- A4T14) on the susceptibility to breast cancer development, also, to figure out their diagnostic and prognostic roles.

Subjects and Methods: Blood samples were obtained from breast cancer patients and controls. Genotyping of CCND1 and p73 genes were carried out by PCR-RFLP and PCR-CTPP; respectively.

Results: In comparison with the control group, CCND1 (G870A) GA and AA genotypes frequencies were significantly higher in breast cancer patients $(\mathrm{p}=0.035$ and $\mathrm{p}=0.002$; respectively), whereas CCND1 (G870A) GG genotype frequency was significantly lower ( $<<$ 0.001). The CCND1 GA and AA genotypes significantly increased the risk for developing breast cancer compared with the GG genotype The CCND1 (GA+AA) genotypes were significantly correlated with disease-free survival (DFS) of breast cancer patients. In comparison with the control group, p73 (G4C14/A4T14) GC/AT and AT/AT genotypes frequencies were significantly higher in breast cancer patients $(\mathrm{p}=0.013$ and $\mathrm{p}=0.04$; respectively), whereas $\mathrm{p} 73$ (G4C14/A4T14) GC/GC genotype frequency was significantly lower ( $\mathrm{p}=0.004)$. Compared with the GC/GC genotype, the p73 GC/AT and AT/AT genotypes significantly increased the risk for developing breast cancer Beside being significantly correlated with DFS, p73 [(GC/AT)+ (AT/AT)] genotypes were indirectly correlated with tumor size, tumor pathological grade, patient's clinical stage, number of axillary lymph node involvement and Her2/neu expression.

Conclusion: The GA and AA genotypes of CCND1 (G870A) polymorphism and the GC/AT and AT/AT genotypes of p73 (G4C14- toA4T14) polymorphism can be used as diagnostic markers in breast cancer patients. The presence of the CCND1 (G870A) GA and AA genotypes and the GC/AT and AT/AT genotypes of p73 (G4C14- to- A4T14) polymorphism can increase the susceptibility to breast cancer incidence. Both of CCND1 (G870A) and p73 (G4C14- to- A4T14) polymorphisms can be used for prognosis of breast cancer patients.
\end{abstract}

Keywords: Breast Cancer; Polymorphism; CCND1 (G870A); P73 (G4C14-to- A4T14); Risk; Diagnosis; Prognosis

\section{Introduction}

Molecular epidemiology is an emerging new field that combines highly sensitive molecular techniques for detecting early damages associated with cancer [1]. Among women; breast cancer remains the most commonly diagnosed cancer. Genetic risk factors contribute to about $5-10 \%$ of all cases, $90-95 \%$ of them result from somatic mutation and about $5-10 \%$ are inherited as a result of germ line mutation in autosomal dominant breast cancer susceptibility genes [2]. It was reported that genetic alterations play a significant role in the development of breast carcinoma [3]. To date, some cancer susceptibility genes have been identified such as cyclin D1 and p73 genes [4].
Cyclin D1, a protein encoded by the CCND1 gene located on chromosome $11 \mathrm{q} 13$, is the major regulatory protein involved in transition of cells from $\mathrm{G} 1$ to the $\mathrm{S}$ phase during cell division. It functions during the G1 stage of the cell cycle by binding to cyclin-dependent kinases 4/6 (CDK 4/6) forming a heterodimeric molecule which is an active protein kinase that phosphorylates specific substrates to modulate the G1/S interphase and promot cell cycle progression to $S$ phase [4]. After entering the $S$ phase, phosphorylation of the cyclin D1 protein at threonine residue-286 enhances its export from the nucleus and subsequent proteolysis, thereby limiting the effect of this protein in promoting cell cycle progression $[5,6]$.

A polymorphism at nucleotide 870 of the CCND1 gene, G870A (CCND1 G870A polymorphism, rs603965), results in an aberrantly spliced cyclin D1 variant (termed cyclin D1b) [7]. This mutant 
transcript differs from the native cyclin D1 isoform (termed cyclin D1a) in the last 55 amino acids of the carboxy terminus, thereby lacking the Thr-286 phosphorylation site required for nuclear export and subsequent proteasome-mediated degradation, leading to an increase in the half-life of the alternate cyclin D1b. This ends with premature cell transition to $S$ phase with propagation of unrepaired DNA damage and accumulation of genetic errors, therefore leading to selective advantage to abnormal cell proliferation [8]. Overexpression of CCND1 disrupts normal cell cycle, possibly promoting cancer development and progression, including breast cancer [9].

A number of studies have linked the CCND1 A allele to increased cancer risk, but the evidence has not been entirely consistent [10], [11]. Few studies have investigated the effect of CCND1 (G870A) polymorphism on cancer prognosis with mixed results $[10,12]$. Lu et al (2009) [13] conducted a meta-analysis on the association between CCND1 (G870A) polymorphism and the risk of breast cancer, and showed that there was an increased risk of breast cancer for carriers of the A allele in Caucasians but not in an Asian population. They suggested that different genetic background and environmental exposures might also contribute to the ethnic difference. Bedewy et al. (2013) [14] studied the association between CCND1 (G870A) polymorphism and the risk of breast cancer in Egyptian female patients and recommended expansion of further studies to include more patients and healthy controls in order to acquire firm data concerning the CCND1 polymorphic variants and evaluate patients' disease free survival and overall survival in relation to the CCND1 polymorphic variants.

p73, a member of p53 family, shares structural and functional similarities to $\mathrm{p} 53$. It is located on chromosome $1 \mathrm{p} 36$, a region frequently deleted in a variety of tumors, indicating that p73 may be a potential tumor suppressor gene [15]. Indeed, p73 is able to activate a set of p53-response genes and induce cell-cycle arrest or apoptosis in response to DNA damage [16]. Two single polymorphisms located at position $4(\mathrm{G}$ to $\mathrm{A}$ ) and 14 (C to $\mathrm{T}$ ) in the 5' untranslated region ( 5 , UTR) of exon 2 of the p73 gene have been identified and named G4C14-A4T14 (rs numbers 2273953 and 1801173). This polymorphism located upstream of the initiating AUG codon of exon 2, a region which may theoretically form a stem-loop structure that could potentially affect gene expression through alteration of the efficiency of translation initiation, thus, it may lead to functional consequence [17].

The association of p73 (G4C14- to- A4T14) polymorphism with cancer risk has been investigated in different studies with different results $[18,19]$. In the Meta analysis performed by Liu et al. (2011) [20] on the association of p73 (G4C14- to- A4T14) polymorphism with cancer risk, the authors reported significantly elevated cancer risks among Caucasians and Asians. When they made subgroup analysis by country, significantly increased cancer risks were found for the Americans and Japanese but not for Chinese. Liu et al. (2011) [20] explained these discrepancies on the basis of the presence of differences in the underlying genetic backgrounds and/or environmental and social factors in the different populations studied. Up to the best of our knowledge, in Egypt no study carried out to investigate the association of p73 (G4C14to- A4T14) polymorphism with breast cancer risk, diagnosis and prognosis.

The aim of the current study was to figure out the role played by the polymorphic variants of CCND1 (G870A) and p73 (G4C14to- A4T14) in the etiology, diagnosis and prognosis of breast cancer in Egyptian females.

\section{Subjects and methods}

Sample size was calculated using NCSS 2000 (Number Cruncher Statistical System) and PASS (Power Analysis and Sample Size) Program. The minimum sample size required was 160 ( 80 for each group) to achieve $80 \%$ power to detect a difference of 1.7 between both groups with estimated group standard deviations of 3.0 and
1.0 and with a significance level (alpha) of 0.05 using a two-sided two-sample t-test.

This case- control study was conducted on 160 females admitted to the Department of Cancer Management \& Research of the Medical Research Institute, Alexandria University, Egypt. They were divided into two groups. Group I (Breast cancer patients group): it included 80 newly diagnosed breast cancer patients of clinical stages II and III, with mean \pm SD age $(52.62 \pm 10.07 \mathrm{yrs})$. Group II (Apparently normal healthy controls): it included 80 female volunteers clinically free from any chronic disease. The controls were matched with the cases for age $(50.15 \pm 9.43 \mathrm{yrs})$, menopausal and socioeconomic status.

Eligibility criteria: cases were newly diagnosed as breast cancer patients of clinical stages II and III before surgery, not receiving chemotherapeutic, immunomodulatory agent or blood transfusion. Controls were free from any chronic disease and matched with the cases for: age, menopausal and socioeconomic status.

Tools of data collection: signed informed consents were collected from all participants prior to their enrollment in the current study. This research protocol was approved by the ethical committee of the Medical Research Institute, Alexandria University, Egypt. All subjects were subjected to the following: full history taking, clinical examination, routine laboratory investigations including complete blood count (CBC), mammography of breast and ultrasonography of abdomen and liver, radiological investigations including $\mathrm{X}$-ray chest, CT scan and bone scan when needed and fine needle aspiration cytology (FNAC) of breast mass to establish the pathological diagnosis in the patients. The clinicopathologic data were obtained from patients'pathology reports. The collected data included tumor size, tumor pathological grade, number of axillary lymph nodes involvement, Her2/neu expression, vascular invasion and status of estrogen receptor (ER) and progesterone receptor (PR). Each breast cancer patient's clinical stage was determined by the oncologist according to the tumor-nodes-metastasis (TNM) classification system [21].

All 80 breast cancer patients were subjected to Modified Radical Mastectomy (MRM) [22], then received adjuvant combination chemotherapy [5-Fluorouracil, Adriamycin and Cyclophosphamide (FAC)] [23] for 6 cycles. After 6 cycles of chemotherapy, patients were evaluated clinically, laboratory and radiologically to estimate their clinical response. The patients were followed up clinically for 50 months for detection of metastasis. An EDTApreserved whole blood sample was obtained from each control and patient before surgery to investigate CCND1 (G870A) and p73 (G4C14- to- A4T14) polymorphism.

\subsection{Extraction of genomic DNA}

Genomic DNA was purified from peripheral whole blood using a ready- for use DNA extraction kit (QIA amp DNA Blood mini kit, Qiagen, Hilden, Germany) according to the producer's protocol.

\subsection{Genotyping of CCND1 (G870A) polymorphism}

Genotyping of CCND1 (G870A) polymorphism was performed by polymerase chain reaction (PCR)-based restriction fragment length polymorphism (RFLP) method. The primers for analysis were [24]: Forward primer: 5' GTGAAGTTCATTTCCAATCCGC-3'; Reverse primer: 5' GGGACATCACCCTCACTTAC-3' (QIAGEN, Germany) to amplify a 167-bp fragment. The PCR reaction was performed according to the method of Wang et al. [24].

For RFLP analysis, each PCR product $(10 \mu \mathrm{l})$ was subjected to 2 units of ScrF1 restriction enzyme (New England, BioLabs Inc, UK) at $37^{\circ} \mathrm{C}$ for 1 hour and separated by electrophoresis on $2 \%$ agarose gel containing $0.5 \mathrm{mg} / \mathrm{ml}$ ethidium bromide and the bands on the gel were visualized using UV Transilluminator. The genotypes were determined as follows: a single 167-bp fragment for the AA genotype, two fragments of 145 and 22 bp for the GG 
genotype, and three fragments of 167,145 , and $22 \mathrm{bp}$ for the GA genotype (Figure 1).

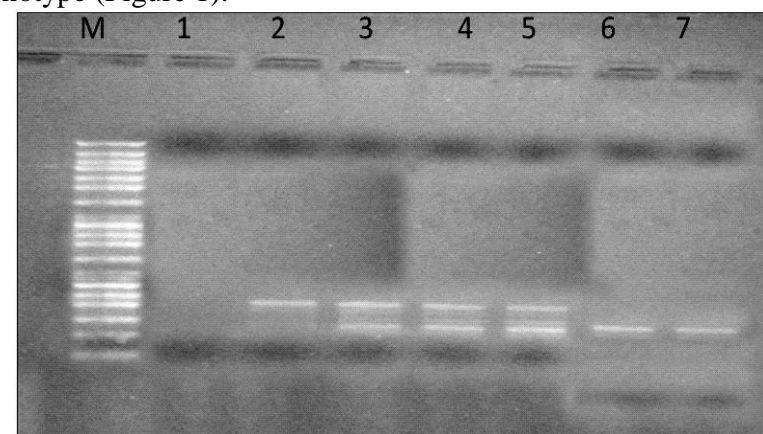

Fig. 1: Representative gel (2\%) for RELP digested PCR products for CCND1 (G870A) genotypes; Lane M is for 50-bp DNA ladder. Lane 2 represents intact PCR products for AA genotype (167- bp); Lanes $(3,4$, and 5) represent restricted PCR products for GA genotype (167-, 145- bp); Lanes $(6,7)$ represent restricted PCR products for GG genotype (145- bp).

\subsection{Genotyping of p73 (G4C14- to- A4T14) polymor- phism}

Genotyping of P73 (G4C14- to- A4T14) polymorphism was carried out using polymerase chain reaction with confronting twopair primers (PCR-CTPP) [25]. The PCR primers were: Forward primer (F1): 5'-CCACGGATGGGTCTGATCC-3'; Reverse primer (R1): 5'-GGCCTCCAAGGGCGACTT-3' and Forward primer (F2): 5'-CCTTCCTTCCTGCAGAGCG-3'; Reverse primer (R2):5'-TTAGCCCAGCGAAGGTGG-3' (QIAGEN, Germany) to amplify a 260 -bp fragment.

The PCR reaction was performed on a thermal cycler (Biometra Tprofessional Thermocycler; Germany). The PCR reaction was carried out in a total volume of $50 \mu \mathrm{l}: 25 \mu \mathrm{l}$ QIAGEN Multiplex PCR Master Mix, $8 \mu \mathrm{l}$ primer mix $(2 \mu \mathrm{l}$ taken from each $20 \mu \mathrm{M}$ primer working solution) and $17 \mu \mathrm{l}(1.4 \mu \mathrm{g})$ template DNA. Each PCR program started with an initial heat-activation step at $95^{\circ} \mathrm{C}$ for $15 \mathrm{~min}$ to activate HotStarTaq DNA Polymerase, followed by 35 cycles of denaturation at $94^{\circ} \mathrm{C}$ for $30 \mathrm{sec}$, annealing at $62^{\circ} \mathrm{C}$ for $90 \mathrm{sec}$, and extension at $72 \mathrm{C}^{\circ}$ for $90 \mathrm{sec}$, with a final extension step at $72{ }^{\circ} \mathrm{C}$ for 10 minutes.

The DNA fragments were separated using electrophoresis on $2 \%$ agarose gel containing $0.5 \mathrm{mg} / \mathrm{ml}$ ethidium bromide and the bands on the gel were visualized by UV Transilluminator. The genotypes were determined as follows: two fragments of (270- bp and 428bp) for the AT/AT genotype, three fragments of (193- bp, 270- bp and 428- bp) for the GC/AT genotype and two fragments of (193$\mathrm{bp}$ and 428- bp) for the GC/GC genotype (Figure 2).

\section{Statistical analysis}

Data were analysed using the Predictive Analytics Software (PASW Statistics 18) for Windows (SPSS Inc, Chicago, USA) The qualitative variables were summarized by frequency and percentage. The Association between categorical variables was tested using Chi-square test. When more than $20 \%$ of the cells have expected count less than 5 , correction for chi-square was conducted using Firsher's exact test.
Odds ratio (OR) and $95 \%$ confidence interval were also calculated for estimation of risk. Univariate survival analysis of the studied parameters was assessed using the Kaplan-Meier method. The results were then compared using the log-rank test in order to determine statistically significant differences between the obtained curves. Results were considered significant at p-value $<0.05$.

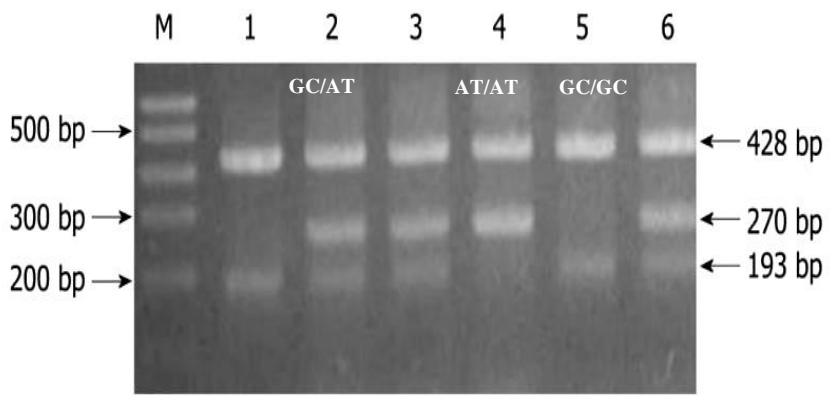

Fig. 2: Representative gel (2\%) by PCR-CTPP for P73 (G4C14-to-A4T14) geneotypes. Lane $\mathrm{M}$ is for 50-bp DNA ladder; Lanes 1,5 for GC/GC genotype with (193-, 428-bp) bands; Lanes 2, 3, 6 for GC/AT genotype with (193-, 270- , 428- bp) bands and Lane 4 for AT/AT genotype with (270-, 428-bp) bands

\section{Results}

Frequency of cyclin D1 (G870A) and p73 (G4C14/A4T14) polymorphic variants in the breast cancer patients and controls.

Cyclin D1 (G870A) GG, GA and AA genotypes frequencies were $63.8 \%, 15 \%$, and $21.2 \%$, respectively, in normal healthy controls and $16.3 \%, 28.7 \%$ and $55.0 \%$, respectively, in breast cancer patients. p73 (G4C14/A4T14) GC/GC, GC/AT and AT/AT genotypes were $75 \%, 18.75 \%$ and $6.25 \%$, respectively, in normal healthy controls and $47.5 \%, 36.2 \%$ and $16.3 \%$, respectively, in breast cancer patients; (Table-I).

For cyclin D1 (G870A) polymorphism, the statistical analysis of these results revealed that, in comparison with the control group, CCND1 (G870A) GA and AA genotypes frequencies were significantly higher in breast cancer patients $(\mathrm{p}=0.035$ and $\mathrm{p}=0.002$; respectively), whereas CCND1 (G870A) GG genotype frequency was significantly lower $(\mathrm{p}<0.001)$. The AA polymorphic variant was of higher sensitivity for prediction of breast cancer than the GA variant (77\% versus $64 \%$ ), while the GA variant was of higher specificity for exclusion of breast cancer than the AA variant (65\% versus $47 \%$ ); (Table-I).

For p73 (G4C14/A4T14) polymorphism, the statistical analysis of these results revealed that, in comparison with the control group, p73 (G4C14/A4T14) GC/AT and AT/AT genotypes frequencies were significantly higher in breast cancer patients $(\mathrm{p}=0.013$ and $\mathrm{p}=0.04$; respectively), whereas $\mathrm{p} 73$ (G4C14/A4T14) GC/GC genotype frequency was significantly lower $(\mathrm{p}=0.004)$. The diagnostic sensitivity of GC/AT and AT/AT genotypes were $43.3 \%$ and $25.5 \%$; respectively and their diagnostic specificity were $80 \%$ and $92.3 \%$; respectively; (Table-I).

Table I: The frequencies, diagnostic sensitivity and specificity of the CCND1 (G870A) and P73 (G4C14/A4T14) polymorphic variants among breast cancer patients and normal healthy controls.

\begin{tabular}{|c|c|c|c|c|c|c|c|c|}
\hline \multicolumn{2}{|c|}{ Polymorphic variants } & \multicolumn{2}{|c|}{$\begin{array}{l}\text { Normal healthy controls } \\
(\mathrm{n}=80)\end{array}$} & \multicolumn{2}{|c|}{$\begin{array}{l}\text { Breast cancer patients } \\
(\mathrm{n}=80)\end{array}$} & \multirow[t]{2}{*}{$\begin{array}{l}\chi^{2} \text { test } \\
(\mathrm{P})\end{array}$} & \multirow[t]{2}{*}{ Sensitivity } & \multirow[t]{2}{*}{ Specificity } \\
\hline & & No. & $\%$ & No. & $\%$ & & & \\
\hline \multirow{3}{*}{ CCND1 (G870A) } & GG & 51 & 63.8 & 13 & 16.3 & $\left(<0.001^{*}\right)$ & - & - \\
\hline & GA & 12 & 15 & 23 & 28.7 & $\left(0.035^{*}\right)$ & $64 \%$ & $65 \%$ \\
\hline & AA & 17 & 21.2 & 44 & 55.0 & $(0.002 *)$ & $77 \%$ & $47 \%$ \\
\hline \multirow{3}{*}{$\begin{array}{c}\text { P73 } \\
(\mathrm{G} 4 \mathrm{C} 14 / \mathrm{A} 4 \mathrm{~T} 14)\end{array}$} & GC/GC & 60 & 75 & 38 & 47.5 & $(0.004 *)$ & - & - \\
\hline & GC/AT & 15 & 18.75 & 29 & 36.2 & $\left(0.013^{*}\right)$ & $43.3 \%$ & $80 \%$ \\
\hline & $\mathrm{AT} / \mathrm{AT}$ & 5 & 6.25 & 13 & 16.3 & $(0.04 *)$ & $25.5 \%$ & $92.3 \%$ \\
\hline
\end{tabular}

*: Statistically significant at $\mathrm{p}<0.05 ; \quad \mathrm{n}$ : sample size 
Table II: Association of CCND1 (G870A) and P73 (G4C14/A4T14) polymorphic variants with breast cancer risk

\begin{tabular}{|c|c|c|c|c|c|c|c|}
\hline \multicolumn{2}{|l|}{ Polymorphic variants } & \multicolumn{2}{|c|}{$\begin{array}{l}\text { Normal healthy controls } \\
(\mathrm{n}=80)\end{array}$} & \multicolumn{2}{|c|}{$\begin{array}{l}\text { Breast cancer patients } \\
(\mathrm{n}=80)\end{array}$} & \multirow[t]{2}{*}{$\begin{array}{l}\text { Test of signif- } \\
\text { icance } \\
\text { (p-value) }\end{array}$} & \multirow[t]{2}{*}{ OR ( $95 \% \mathrm{CI})$} \\
\hline & & No. & $\%$ & No. & $\%$ & & \\
\hline \multirow[t]{2}{*}{ CCND1 (G870A) } & GA & 12 & 15 & 23 & 28.7 & \multirow{2}{*}{$\begin{array}{l}\left(<0.001^{*}\right) \\
\left(<0.001^{*}\right)\end{array}$} & $7.5(2.98-18.99)$ \\
\hline & AA & 17 & 21.2 & 44 & 55.0 & & $10.15(4.44-23.22)$ \\
\hline \multirow{3}{*}{$\begin{array}{c}\text { P73 } \\
\text { (G4C14/A4T14) }\end{array}$} & $\mathrm{GC} / \mathrm{GC}$ & 60 & 75 & 38 & 47.5 & \multirow{3}{*}{$\begin{array}{l}\left(0.003^{*}\right) \\
\left(0.013^{*}\right)\end{array}$} & 1.00 (reference) \\
\hline & GC/AT & 15 & 18.75 & 29 & 36.2 & & $3.05(1.45-6.42)$ \\
\hline & $\mathrm{AT} / \mathrm{AT}$ & 5 & 6.25 & 13 & 16.3 & & $4.11(1.35-12.44)$ \\
\hline
\end{tabular}

*: Statistically significant at $\mathrm{p}<0.05 ; \mathrm{n}$ : sample size; ®: reference genotype; OR: odd's ratio

Association of cyclin D1 (G870A) and p73 (G4C14/A4T14) polymorphic variants with breast cancer risk

Table-II shows the effect of CCND1 (G870A) and p73 (G4C14/A4T14) polymorphisms on the risk for breast cancer development. The CCND1 (G870A) GA and AA genotypes significantly increased the risk for developing breast cancer compared with the GG genotype [OR=7.5, 95\% CI: 2.98- 18.99; $\mathrm{p}<0.001$ and $\mathrm{OR}=10.15,95 \% \mathrm{CI}: 4.44-23.22 ; \mathrm{p}<0.001$; respectively]. The p73 (G4C14/A4T14) GC/AT and AT/AT genotypes significantly increased the risk for developing breast cancer compared with the GC/GC genotype $[\mathrm{OR}=3.05 ; 95 \% \mathrm{CI}: 1.45-6.42 ; \mathrm{p}=0.003$ and $\mathrm{OR}=4.11,95 \% \mathrm{CI}: 1.35-12.44 ; \mathrm{p}=0.013$; respectively].

Correlation of cyclin D1 (G870A) and p73 (G4C14/A4T14) polymorphic variants with clinicopathological data of breast cancer patients

Correlation of different clinicopathological data with breast cancer patients carrying the A allele (AA+GA genotypes) of CCND1 (G870A) polymorphism were non-significant ( $p>0.05$ ). However, breast cancer patients carrying the AT allele (GC/AT+AT/AT genotypes) of p73 (G4C14/A4T14) polymorphism showed significant indirect correlations with tumor size $(\mathrm{r}=-0.75 ; \mathrm{p}=0.032)$, tumor pathological grade $(r=-0.63 ; p=0.021)$, patient's clinical stage $(r=$ $-0.43 ; \mathrm{p}=0.04)$, number of axillary lymph node involvement $(\mathrm{r}=-0.37$; $\mathrm{p}=0.025)$ and Her $2 /$ neu expression $(\mathrm{r}=-0.51 ; \mathrm{p}=0.019)$; all of which were makers of poor prognosis. However; the correlations of p73 polymorphism with vascular invasion, estrogen and progesterone receptors status were non-significant ( $\mathrm{p}>0.05)$.

Correlation of cyclin D1 (G870A) and p73 (G4C14/A4T14) polymorphic variants with disease-free survival of breast cancer patients

Kaplen-Meir disease-free survival (DFS) curve was constructed to study this correlation (figures 3 and 4). Breast cancer patients were followed-up for a median period of 30 months (range 20-50 months) for detection of any metastasis or local recurrence. For CCND1 (G870A) polymorphism, the DFS of breast cancer patients carrying the A allele (i.e., GA+AA genotypes) was compared with those not carrying the A allele (i.e., GG genotype). The median $($ mean \pm SE) DFS was $24.0(23.14 \pm 1.30)$ months for patients with the GG genotype and 44.0(41.92 \pm 1.20$)$ months for patients carrying $(\mathrm{GA}+\mathrm{AA})$ genotypes. The statistical analysis of these data showed that breast cancer patients carrying the A allele had DFS time significantly longer than those not carrying the A allele $(\mathrm{p}<0.001)$. For $\mathrm{p} 73$ (G4C14/A4T14) polymorphism, the DFS of breast cancer patients carrying the AT allele (i.e., GC/AT+AT/AT genotypes) was compared with those not carrying the AT allele (i.e., GC/GC genotype). The median (mean $\pm \mathrm{SE}$ ) DFS was $24.0(24 \pm 1.13)$ months for patients with the GC/GC genotype and 40.0(41.33 \pm 1.45$)$ months for patients with the (GC/AT+AT/AT) genotypes. The statistical analysis of these data showed that breast cancer patients carrying the AT allele had DFS time significantly longer than those not carrying the AT allele $(\mathrm{p}<0.001)$; Table-III.

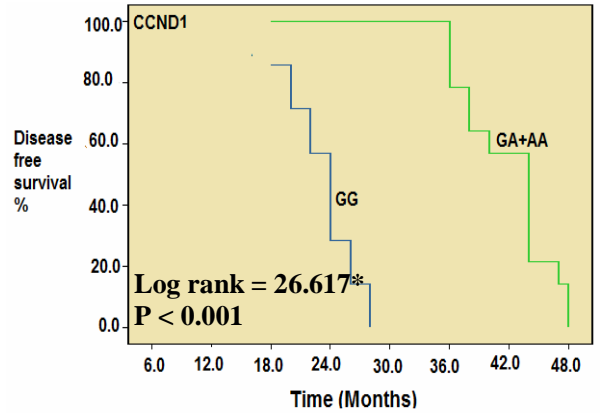

Fig. 3: Kaplan-Meier disease-free survival for breast cancer patients carrying the GG or (GA+AA) genotypes of CCND1 (G870A) polymorphism.

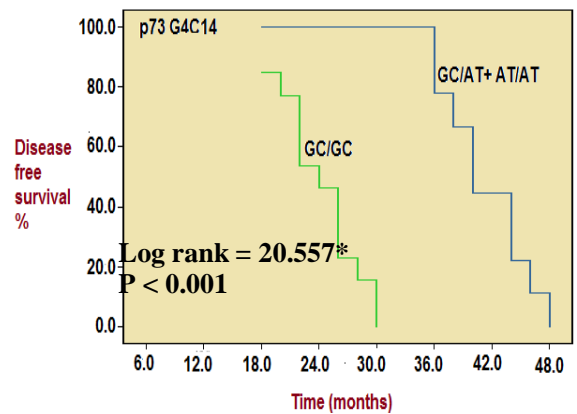

Fig. 4: Kaplan-Meier disease free survival for breast cancer patients carrying the GC/AT or (GC/AT+AT/AT) genotypes of P73 (G4C14/A4T14) polymorphism.

Table III: Association of CCND1 (G870A) and P73 (G4C14/A4T14) genotypes with breast cancer disease-free survival (DFS)

\begin{tabular}{lllll}
\hline Genotype & & $\begin{array}{l}\text { Metastasis } \\
\mathrm{n}=22\end{array}$ & $\begin{array}{l}\text { Non Metastasis } \\
\mathrm{n}=58\end{array}$ & $\begin{array}{l}\text { Median (Mean } \pm \text { SE) } \\
\text { DFS (months) }\end{array}$ \\
\hline \multirow{2}{*}{ CCND1 G870A } & GG (-ve) $(\mathrm{n}=13)$ & $7(53.9 \%)$ & $6(46.2 \%)$ & $24.0(23.14 \pm 1.30)$ \\
& (GA+ AA) $(+\mathrm{ve})(\mathrm{n}=67)$ & $15(22.4 \%)$ & $52(77.6 \%)$ & $44.0(41.92 \pm 1.20)$ \\
p73 & GC/GC (-ve) $(\mathrm{n}=38)$ & $13(34.2 \%)$ & $25(65.8 \%)$ & $24.0(24 \pm 1.13)$ \\
(G4C14/A4T14) & [(GC/AT)+(AT/AT) & $9(21.4 \%)$ & $33(78.6 \%)$ & $40.0(41.33 \pm 1.45)$
\end{tabular}

\footnotetext{
*: Statistically significant at $\mathrm{p}<0.05 ; \quad \mathrm{n}$ : sample size; $\quad$ DFS: disease-free survival
} 


\section{Discussion}

In the present study, in comparison with the control group, CCND1 (G870A) GA and AA genotypes frequencies were significantly higher in breast cancer patients, whereas the GG genotype frequency was significantly lower, suggesting that CCND1 (G870A) GA and AA genotypes may play a role in the susceptibility to breast cancer and can be used to differentiate between breast cancer patients and normal controls.

In agreement with our results, Bedewy et al. (2013) [14] found that the frequency of having the GG genotype was higher in the control group than in breast cancer patients when compared with the distribution of the other genotypes. Furthermore, they found a significant association between the A allele either in heterozygous (GA) or homozygous state (AA) and breast cancer patients. Also, Yu et al. (2008) [12] found that the frequency of GG genotype was higher in the control group, while the frequency of A allele either in homozygous (AA) or heterozygous (GA) state was higher in breast cancer patients. On the other hand, Krippl et al (2003) [10] reported that CCND1 genotypes frequencies were similar among breast cancer patients and controls.

In the present study, it was found that individuals carrying the GA and AA genotypes of CCND1 (G870A) polymorphism had 7.5 and 10.15 fold increased risk for the development of breast cancer compared with those carrying the GG genotype. Bedewy et al. (2013) [14] reported a positive risk of developing breast cancer for those having the A allele either in homozygous (AA) or heterozygous (GA) state when compared to GG genotype. Shu et al.(2005) [9] showed that the A allele, either in homozygous (AA) or heterozygous (GA) state when compared to GG genotype was only weakly associated (borderline significance) with the risk of breast cancer. This shows a possible oncogenic effect for the A allele which is maintained in both homozygous and heterozygous states.

Betticher et al (1995) [7] reported that individuals carrying the AA genotype produced an altered transcript-b of CCND1 that may have longer half-life. Therefore cells with damaged DNA carrying the A allele of CCND1 (G870A) polymorphism may bypass the G1/S phase check point easily compared with those not carrying this polymorphism.

Sawa et al (1998) [26] demonstrated that high level of normal transcript-a inhibits entry into and completion of the $\mathrm{S}$ phase of the cell cycle. These observations suggest that the genotyping difference of CCND1 (G870A) polymorphism may influence the biological behavior of cells, thus altering the risk of developing breast cancer by producing different transcripts of CCND1.

$\mathrm{Yu}$ et al (2008) [12] indicated that CCND1 (G870A) polymorphism makes a significant contribution to breast cancer in China with preponderance of breast cancer in young women. Their results showed that the GA and AA subgroups were at increased risk for developing breast cancer compared with those with the GG genotype. The results of the current study were in complete agreement with those of $\mathrm{Yu}$ et al. (2008) [12] regarding the breast cancer risk provided by the GA and AA alleles. Krippl et al. (2003) [10], showed that no risk of developing breast cancer for those having the A allele either in homozygous (AA) or heterozygous (GA) state.

In the study of Onay et al. (2008) [11], homozygosity for the A allele (AA), when compared to GG genotype, showed a positive risk of developing breast cancer in both the Ontario and the Finland samples. However, the presence of the A allele (i.e.; AA+GA genotypes) when compared to GG genotype showed no positive risk association in both Ontario and Finland populations.

In the current study, it was found that carrying one A allele (i.e. GA or AA genotype) of CCND1 (G870A) polymorphism was sufficient to increase the risk for breast cancer occurrence. So, we combined the GA and AA genotypes (GA+AA) of CCND1 and considered them representing the abnormal genotypes, while the GG genotype representing the normal genotype. In the current study, there was no significant correlation of carrying the A allele of CCND1 (G870A) polymorphism with breast cancer clinicopathological features. Yoylim-Eraltan et al. (2009) [27] reported that the distribution of CCND1 (G870A) polymorphic variants were non-significantly different among stages T3-T4 and T1-T2 tumors and also not associated with the number of axillary lymph node involvement. Also, Abramson et al. (2010) [28] found that cyclin D1b expression did not significantly correlate with any adverse prognostic marker of breast cancer patients.

In the present study, it was found that carrying the A allele of CCND1 (G870A) polymorphism (i.e. AA+GA genotypes) was significantly correlated with longer DFS for breast cancer than did patients not carrying the A allele (GG genotype). The favorable DFS for breast cancer patients carrying the A allele despite its positive association with increased risk of breast cancer development could be attributed to the induction of cyclin D1 degradation by chemotherapy causing cell death and apoptosis [29]. In concordance with our findings, Shu et al. (2005) [9] found that carrying the A allele of the CCND1 (G870A) polymorphism was related to a favorable outcome for breast cancer, particularly among those with a stage III or IV or ER/PR- negative breast cancer. Shu et al. (2005) [9] findings were in agreement with earlier observations that CCND1 induces apoptosis [30]. Because rapidly proliferating breast cancer is likely to be more sensitive to chemotherapy (11), Shu et al. (2005) [9] finding of a strong inverse association of CCND1 (G870A) polymorphism with breast cancer survival among women with late stage or ER/PR- negative breast cancer may be a result of increased response to chemotherapy due to the increased apoptosis associated with the CCND1 (G870A) polymorphism and increased proliferation associated with advanced disease characteristics. Shu et al. (2005) [9] also reported that it is possible that among patients with ER-positive breast cancer, the possible beneficial effect of CCND1 on prognosis may be offset by its ability to bind to the ER, compromising the effect of hormonal therapy. The shorter DFS of breast cancer patients carrying GG genotype of CCND1 (G870A) can be explained by the study of Yoylim-Eraltan et al (2009) [27] who found that breast cancer patients carrying the GG genotype of CCND1 (G870A) polymorphism had a 1.7 fold increased risk of axillary lymph node metastasis compared with those with the AA and GA genotypes.

On the other hand, a previous study on CCND1 (G870A) polymorphism and breast cancer survival reported a null association [31]. The inconsistent literature on CCND1 (G870A) polymorphism and cancer prognosis may be attributable to the characteristics of cancers, study setting and design, the treatment regimens, inappropriate controls (convenience samples such as lab personnel or hospital controls with other diseases) and failure to control for ethnicity $[9,19]$.

In the present study, in comparison with the control group, p73 (G4C14/ A4T14) GC/AT and AT/AT genotypes frequencies were significantly higher in breast cancer patients, whereas the GC/GC genotype frequency was significantly lower, suggesting that $\mathrm{p} 73$ (G4C14/ A4T14) GC/AT and AT/AT genotypes may play a role in the etiology of breast cancer. Li et al. (2004) [32] showed that the frequencies of the AT allele and genotypes of p73 (G4C14/A4T14) polymorphism were significantly more common in cases with squamous cell carcinoma of head and neck (SCCHN) than in cancer- free controls.

In the present study, it was found that individuals carrying the GC/AT and AT/AT genotypes of p73 (G4C14/A4T14) polymorphism had 3.05 and 4.11 fold increased risk for the development of breast cancer compared with those carrying the GC/GC genotype. This means that the presence of one AT allele (i.e. GC/AT or AT/AT genotype) of p73 (G4C14/A4T14) polymorphism was sufficient to increase the risk of breast cancer occurrence. Although how this p73 (G4C14/A4T14) polymorphism influences the development of breast cancer is unknown, one possible explanation is that the GC to AT change may lead to formation of a stem loop structure and so may influence the translation efficiency of p73 gene [33]. Another possibility is that this p73 polymorphism is in linkage disequilibrium with other functional polymorphisms that affect either the expression or activity levels of enzymes involved in tumorigenesis [34]. The association of p73 (G4C14/ A4T14) polymor- 
phism with the risk of cancer development has been investigated by many epidemiological studies; however, the results were conflicting. Liu et al. (2011) (20) found that individuals with the AT/AT genotype were significantly associated with an increased cancer risk than those with GC/AT and/or GC/GC genotypes. Li et al. (2004) [32] found that Chinese women with the GC/GC genotype had an increased risk of developing breast cancer than those with the GC/AT or AT/AT genotype. On the other hand, Huang et al. (2003) [19] reported no association between breast cancer risk and p73 (G4C14/ A4T14) polymorphism.

In our study, it was found that the presence of one AT allele (i.e. GC/AT or AT/AT genotype) of p73 (G4C14/ A4T14) polymorphism was sufficient to increase the risk for breast cancer occurrence. So, in the current study, we combined the GC/AT and AT/AT genotypes (GC/AT+AT/AT) of p73 (G4C14/A4T14) polymorphism and considered them representing the abnormal genotypes, while the GC/GC genotype representing the normal genotype.

In the present study, the combined variants $(\mathrm{GC} / \mathrm{AT}+\mathrm{AT} / \mathrm{AT})$ of p73 (G4C14/A4T14) polymorphism were indirectly correlated with clinicopathological data of breast cancer except vascular invasion, PR and ER status. Our results confirmed those reported by Xin and Chengyi (2012) [35] who concluded that breast cancer patients carrying GC/GC genotype may have bad prognosis. The finding of Xin and Chengyi (2012) [35] was also supported by our results regarding the longer DFS of patients carrying the AT allele of p73 (G4C14/A4T14) polymorphism (i.e.; GC/AT + AT/AT genotypes) compared with those not carrying the AT allele (i.e.; GC/GC genotype). Previous studies have shown that overexpression of $\mathrm{p} 73$ protein is a poor prognostic factor in breast cancer patients [36]. Daniel et al. (2000) [37] found that the AT allele leads to a slight tendency towards lower expression of the p73 protein in tumor, which might be an explanation for the better DFS in the AT allele carrying patients. A possible explanation for the tendency towards less protein expression with AT/AT homozygotes might be that the change from GC to AT may lead to the formation of a stem loop structure, thus, influencing the translation efficiency of p73 in tumors. Unexpectedly, our results taken together seem to show that there was a higher risk of developing breast cancer in females carrying the AT allele, but once affected, the patients have a better disease free survival.

$\mathrm{Li}$ et al. (2004) [32] demonstrated that the GC/GC genotype was strongly correlated with an unfavorable DFS or overall survival of breast cancer patients. In their study, the GC/GC genotype was correlated with a positive axillary lymph- node status, suggesting that breast cancer patients with the GC/GC genotype had a more aggressive phenotype.

Finally from the results of current study, it could be concluded that homozygosity or heterozygosity for the A allele of CCND1 (G870A) and AT allele of p73 (G4C14-to- A4T14) polymorphism can be used for diagnosis of Egyptian breast cancer patients. The presence of one A allele of CCND1 (G870A) polymorphism and the presence of one AT allele of p73 (G4C14-to- A4T14) polymorphism are sufficient to increase the risk for breast cancer development. In case of homozygosity, the risks significantly increased. The combined variants (GC/AT+AT/AT) of p73 (G4C14to- A4T14) polymorphism and the combined variants (GA+AA) of CCND1 (G870A) polymorphism can be used for prognosis and prediction of the clinical outcome of breast cancer patients.

\section{References}

[1] Lee K-M, Han S, Park W-Y, Kang D. Identification and application of biomarkers in molecular and genomic epidemiologic research. J Prev Med Public Health 2009; 42: 349-55. http://dx.doi.org/10.3961/ipmph.2009.42.6.349.

[2] Kwan ML, Kushi LH, Weltzien E, Maring B, Kutner SE, Fulton $\mathrm{RS}$, et al. Epidemiology of breast cancer subtypes in two prospective cohort studies of breast cancer survivors. Breast Cancer Res 2009; 1: R31-43. http://dx.doi.org/10.1186/bcr2261.

[3] Sadikovic B, Al-Romaih K, Squire JA, Zielenska M. Cause and consequences of genetic and epigenetic alterations in human can- cer. Curr Genomics 2008;

http://dx.doi.org/10.2174/138920208785699580.

9:394-408

[4] Sherr CJ. D-type cyclins. Trends Biochem Sci 1995; 20:187-90. http://dx.doi.org/10.1016/S0968-0004(00)89005-2.

[5] Lin DI, Barbash O, Kumar KGS, Weber JD, Harper JW, KleinSzanto AJP, et al. Phosphorylation-dependent ubiquitination of cyclin D1 by the SCF (FBX4-alphaB crystallin) complex. Mol Cell 2006; 24:355-66. http://dx.doi.org/10.1016/i.molcel.2006.09.007.

[6] Diehl JA, Zindy F, Sherr CJ. Inhibition of cyclin D1 phosphorylation on threonine-286 prevents its rapid degradation via the ubiquitin-proteasome pathway. Genes Dev. 1997; 11:957-72. http://dx.doi.org/10.1101/gad.11.8.957.

[7] Betticher DC, Thatcher N, Altermatt HJ, Hoban P, Ryder WD, Heighway J. Alternate splicing produces a novel cyclin D1 transcript. Oncogene 1995; 11:1005-11.

[8] Solomon DA, Wang Y, Fox SR, Lambeck TC, Giesting S, Lan Z, et al. Cyclin D1 splice variants. Differential effects on localization, RB phosphorylation, and cellular transformation. J Biol Chem 2003; 278:30339-47. http://dx.doi.org/10.1074/jbc.M303969200.

[9] Shu XO, Moore DB, Cai Q, Cheng J, Wen W, Pierce L, et al. Association of cyclin D1 genotype with breast cancer risk and survival. Cancer Epidemiol Biomarkers Prev 2005; 14: 91-7.

[10] Krippl P, Langsenlehner U, Renner W, Yazdani-Biuki B, Wolf G, Wascher TC, et al. The G870A polymorphism of the cyclin D1 gene is not associated with breast cancer. Breast Cancer Res Treat 2003; 82: 165-8. http://dx.doi.org/10.1023/B:BREA.0000004372.20461.33.

[11] Onay UV, Aaltonen K, Briollais L, Knight JA, Pabalan N, Kilpivaara O, et al. Combined effect of CCND1 and COMT polymorphisms and increased breast cancer risk. BMC Cancer 2008; 8 6. http://dx.doi.org/10.1186/1471-2407-8-6.

[12] Yu CP, Yu JC, Sun CA, Tzao C, Ho JY, Yen AM. Tumor susceptibility and prognosis of breast cancer associated with the G870A polymorphism of CCND1. Breast Cancer Res Treat 2008; 107: 95102. http://dx.doi.org/10.1007/s10549-007-9522-y.

[13] Lu C, Dong J, Ma H, Jin G, Hu Z, Peng Y, et al. CCND1 (G870A) polymorphism contributes to breast cancer susceptibility: a metaanalysis. Breast Cancer Res Treat 2009; 116: 571-5 http://dx.doi.org/10.1007/s10549-008-0195-y.

[14] Bedewy AML, Mostafa MH, Saad AA, EL-Maghraby SM, Bedewy MML, Hilal AM, et al. Association of Cyclin D1 A870G polymorphism with two malignancies: Acute lymphoblastic leukemia and breast cancer. J BUON 2013; 18: 227-38

[15] Moll UM, Slade N. P63 and $p 73$ : roles in development and tumor formation. Mol Cancer Res 2004; 2: 371-86.

[16] Jost C A, Marin M C, Kaelin W G. P73 is a simian p53-related protein that can induce apoptosis. Nature 1997; 389: 191-4. http://dx.doi.org/10.1038/38298.

[17] De Feo E, Persiani R, La Greca A, Amore R, Arzani D, Rausei S, et al. A case-control study on the effect of p53 and p73 gene polymorphisms on gastric cancer risk and progression. Mutat Res 2009; 30: 60-5. http://dx.doi.org/10.1016/j.mrgentox.2009.02.009.

[18] Niwa Y, Hirose K, Matsuo K, Tajima K, Ikoma Y, Nakanishi T. Association of $p 73$ (G4C14-to-A4T14) polymorphism at exon 2 and p53 (Arg72Pro) polymorphism with the risk of endometrial cancer in Japanese subjects. Cancer Lett 2005; 219: 183-90. http://dx.doi.org/10.1016/j.canlet.2004.10.018.

[19] Huang XE, Hamajima N, Katsuda N, Matsuo K, Hirose K, Mizutani M. et al. Association of p53 codon Arg72Pro and p73 G4C14 to-A4T14 at exon 2 genetic polymorphisms with the risk of Japanese breast cancer. Breast Cancer 2003; 10: 307-11. http://dx.doi.org/10.1007/BF02967650.

[20] Liu F, Liu L, Li B, Wei Y, Yan L, Wen T, et al. p73 (G4C14A4T14) polymorphism and cancer risk: a meta-analysis based on 27 case-control studies. Mutagenesis 2011; 26:573-81. http://dx.doi.org/10.1093/mutage/ger018.

[21] Haskell CM, Lowitz BB, Casciato AD. Breast cancer. In: Casciato $\mathrm{AD}$ and Lowitz BB; eds. Manual of clinical oncology; $2^{\text {nd }}$ ed. little and Brown Company, Boston, Toronto (pub.) 1985, pp. 150-65.

[22] Rintoul RF. Operations on the breast. In: Farquhaerison,s text book of operative surgery; 7 th ed. Churchill living stone (pub.) 1986, pp. 270-81.

[23] Abeloff MD, Lichter AS, Niederhuber JE, Pierce LJ and Aziz DC. Breast. In: Abeloff MD, Armitage JO, Licher AS, Niederhuber JE; eds. Clinical Oncology, Churchil living stone Inc. (Pub.), 1995, Chapter 73, pp 1617-714

[24] Wang L, Habuchi T, Takahashi T, Mitsumori K, Kamoto T, Kakehi $\mathrm{Y}$, et al. Cyclin D1 gene polymorphism is associated with an increased risk of urinary bladder cancer. Carcinogenesis 2002; 23 257-64. http://dx.doi.org/10.1093/carcin/23.2.257. 
[25] Hamajima N, Tamakoshi A, Kawase H, Wakai K, Katsuda N, Saito $\mathrm{T}$, et al. Duplex polymerase chain reaction with confronting twopair primers (PCR-CTTP) for genotyping alcohol dehydrogenase â subunit $(\mathrm{ADH} 2)$ and aldehyde dehydrogenase 2(ALDH2). Alcohol \& Alcoholism 2003; 38: 407-10 http://dx.doi.org/10.1093/alcalc/agg096.

[26] Sawa H, Ohshima TA, Ukita H, Murakami H, Chiba Y, Kamada H, et al. Alternatively spliced forms of Cyclin D1 modulate entry into the cell cycle in an inverse manner. Oncogene 1998; 16: 1701-12. http://dx.doi.org/10.1038/sj.onc.1201691.

[27] Yoylim-Eraltan I, Ergen A, Gormus U, Arikan S, Kucucuk S, Sahin $\mathrm{O}$, et al. Breast cancer and cyclin D gene polymorphism in Turkish women. In vivo 2009; 23:767-72.

[28] Abramson VG, Troxel AB, Feldman M, Mies C, Wang Y, Sherman $\mathrm{L}$, et al . Cyclin D1b in human breast carcinoma and coexpression with cyclin D1a is associated with poor outcome. Anticancer Res 2010; 30:1279-85.

[29] Zhou Q, Hopp T, Fuqua SA, Steeg PS. Cyclin D1 in breast premalignancy and early breast cancer: implications for prevention and treatment. Cancer Lett 2001; 162:3 - 17. http://dx.doi.org/10.1016/S0304-3835(00)00657-1.

[30] Lu F, Gladden AB, Diehl JA. An alternatively spliced cyclin D1 isoform, cyclin D1b, is a nuclear oncogene. Cancer Res 2003; 63: 7056-61.

[31] Grieu F, Malaney S, Ward R, Joseph D, Iacopetta B. Lack of association between $C C N D 1$ (G870A) polymorphism and the risk of breast and colorectal cancers. Anticancer Res 2003; 23: 4257-9.

[32] Li G, Sturgis EM, Wang L, Chamberlain RM, Amos CI, Spitz MR, et al. Association of a $p 73$ exon 2 G4C14-to-A4T14 polymorphism with risk of squamous cell carcinoma of the head and neck. Carcinogenesis 2004; 25 : 1911-6. http://dx.doi.org/10.1093/carcin/bgh197.

[33] Hishida A, Matsuo K, Tajima K, Ogura M, Kagami Y, Taji H. Polymorphisms of p53 Arg72Pro, p73 G4C14-to-A4T14 at exon 2 and p21 Ser31Arg and the risk of non-Hodgkin's lymphoma in Japanese. Leuk Lymphoma 2004; 45: 957-64. http://dx.doi.org/10.1080/10428190310001638878.

[34] Ryan BM, McManus R, Daly JS, Carton E, Keeling PW, Reynolds JV. A common $p 73$ polymorphism is associated with a reduced incidence of esophageal carcinoma. Br J Cancer 2001; 85; 1499-503. http://dx.doi.org/10.1054/bjoc.2001.2066.

[35] Xin Z, Chengyi WU. p73 polymorphisms and clinicopathologic characteristics in breast cancer. J Cent South Univ (Med Sci) 2012; 37: 238-43.

[36] Du CW, Kimijima I, Otake T, Abe R, Takenoshita S, Zhang GJ Down-regulation of $p 73$ correlates with high histological grade in Japanese with breast carcinomas. Chin Med J (Engl). 2011; 124:2275-8.

[37] Daniel Z, Tschan MP, Grob TJ, Peters UR, Fink D, Haenggi W, et al. Differential expression of $p 73$ splice variants and protein in benign and malignant ovarian tumors. Int $\mathrm{J}$ Cancer 2000; 88: 66-70. http://dx.doi.org/10.1002/1097-0215(20001001)88:1<66::AID-

IJC10>3.0.CO;2-Y. 